\title{
Role of Chest Radiographs in COVID-19 Positive Patients in a Single Institute in Central India
}

\author{
Dr. Vaishali Kundu ${ }^{1}$, Dr. Krati Jain ${ }^{1}$, Dr. Manish Bhagat ${ }^{2 *}$
}

${ }^{1}$ Resident, Department of Radiodiagnosis, SAIMS Indore, Madhya Pradesh, India

${ }^{2}$ Associate Professor\& HOD, Department of Radiodiagnosis, SAIMS Indore, Madhya Pradesh, India

*Corresponding author: Dr. Manish Bhagat

Aims and Objectives: To describe the spectrum and severity of findings of COVID-19 pneumonia on chest radiography in patients who have tested positive on real time polymerase chain reaction (RT-PCR) in a single Institute in Central India during a span of three months. Results: Most common chest radiographic findings in COVID-19 patients were ground glass opacities, consolidation and hilar lymphadenopathy and pathological chest radiographs were more common among patients with comorbidities. Conclusion: Through this study we have tried to highlight the importance of chest radiography especially portable radiography in inpatient settings in detecting the degree of lung involvement in patients with or without comorbidities.

Keywords: Consolidation, chest radiograph (CXR), pneumonia, COVID-19(corona virus), real time polymerase chain reaction $(\mathrm{RT}-\mathrm{PCR})$.

Copyright $\odot$ (C)21 The Author(s): This is an open-access article distributed under the terms of the Creative Commons Attribution 4.0 International License (CC BY-NC 4.0) which permits unrestricted use, distribution, and reproduction in any medium for non-commercial use provided the original author and source are credited.

\section{INTRODUCTION}

Cases of viral pneumonia with an unknown origin emerged from Wuhan, China, and were registered to the World Health Organization (WHO) on December 31, 2019 [1]. On January 7, 2020, the 2019 novel coronavirus strain (2019-nCoV; later renamed severe acute respiratory syndrome coronavirus 2 [SARSCoV-2]) was confirmed as the primary etiology of these reported cases, and the outbreak was subsequently labelled as the coronavirus disease (COVID19) [2]. On 30th January 2020, COVID-19 was declared as a global health emergency by the World Health Organization [3].

Information on COVID-19 is still evolving and a detailed understanding of the diagnostic imaging hallmarks, atypical features, and evolution of chest imaging findings is essential for effecient patient management and treatment.

The present COVID-19 radiological literature is primarily focused on CT findings [4-9], which is much more sensitive than chest X-ray (CXR). In China, CT was primarily the first-line investigation for COVID- 19 [10-11]. However, this led to a huge burden on radiology departments and caused an immense challenge for infection control in the CT room and console. Few medical centers in China dedicated specific CT scanners for examining suspected COVID19 patients only, a practice being instituted with difficulty in the United Kingdom. The American College of Radiology(ACR) postulates that CT decontamination required after scanning COVID-19 patients leads to disruption of radiological service availability, and tells that portable chest $\mathrm{x}$-ray (CXR) may be considered to minimize the risk of crossinfection [12]. Italian and British hospitals are starting to employ CXR as a first-line triage tool due to long real time polymerase chain reaction (RT-PCR) turnaround times. In our institute located in central India, we have thoroughly studied chest radiographs taken in patients admitted during a span of three months (who tested positive for COVID 19 with RTPCR) and was used as the primary first line imaging investigation.

Our study aims to highlight the importance of portable chest radiography in admitted patients with COVID19 pneumonia especially in detecting the presence of consolidation, ground glass opacities, hilar lymphadenopathy and pleural effusion.

\section{Materials And Methods}

A Retrospective study was done in Sri Aurobindo Medical College and PG Institute, Indore, Madhya Pradesh, India and was approved by the 
hospital ethical committee. The study was done of COVID-19 patients with RT-PCR confirmation and CXRs of these were evaluated between the months of May 2020 and July 2020. Baseline and serial CXRs were reviewed along with RT-PCRs.

550 patients were included in this study of which 50 were lost due technical reasons and error in data acquisition, so a total of 500 patient's CXRs were analysed for the presence of consolidation (bilateral or unilateral), patchy ground glass opacities, hilar lymphadenopathy, pleural effusion.

We also analysed the chest radiographs to look for the impact of disease in patients with and without comorbidities like diabetes mellitus, hypertension, asthma, pulmonary tuberculosis and malignancy. Age factor was also taken into consideration.

These 500 patients had subsequent radiographs taken at day 10 of positive RTPCR test and were compared with the baseline radiographs.

\section{Image Acquisition \& Analysis}

All CXRs were acquired as computed or digital radiographs following usual local protocols. We used GE Brivo XR115 mobile xray system to do all the CXRs. All were acquired in the posteroanterior (PA) or anteroposterior (AP) projection. Almost all of the CXRs at admission were performed in the PA projection with a few in AP projections that were in the Critical care units. All follow-up CXRs were performed in the AP projection. The entire chest $\mathrm{X}$ rays were analysed by three experienced radiologists having adequate training in reading chest radiographs.

\section{RESULTS}

Table-1: Patient characteristics

\begin{tabular}{|l|l|}
\hline Parameter & $\begin{array}{l}\text { No. of patients(n = 500) } \\
\text { and their percentages }\end{array}$ \\
\hline Male gender & $236(47.2 \%)$ \\
\hline Female gender & $264(52.8 \%)$ \\
\hline Age (in years) & $54+/-18$ \\
\hline Diabetes mellitus & $75(15 \%)$ \\
\hline Hypertension & $85(17 \%)$ \\
\hline Asthma & $17(3.4 \%)$ \\
\hline Malignancy & $11(2.2 \%)$ \\
\hline Pulmonary tuberculosis & $12(2.4 \%)$ \\
\hline
\end{tabular}

CXRs of COVID-19 patients with RT-PCR confirmation admitted in our hospital during a span of three months were analysed.

CXRs were done of 550 patients of which 50 CXR were lost due to faulty techniques and errors during data acquisition. CXRs of 500 patients were properly analysed and patient characteristics were also taken into consideration (summarized in table 1).

ut of 500 patients, 236(47.2\%) were male and $264(52.8 \%)$ were female. Mean age of patients was 54 years. Co-morbidities were present in 200(40\%) patients including diabetes mellitus (15\%), hypertension $(17 \%)$, asthma $(3.4 \%)$, pulmonary tuberculosis $(2.4 \%)$ and malignancy $(2.2 \%)$ were taken into consideration.

These 500 patients had follow up CXRs done at day 10 of testing positive on RTPCR for COVID 19 virus. Major Xray findings have been summarized in table 2 .

Table-1: Major Xray findings

\begin{tabular}{|l|l|}
\hline Parameter & $\begin{array}{l}\text { No. of patients(n= 500) and } \\
\text { their percentages }\end{array}$ \\
\hline No. of normal chest Xrays & $120(24 \%)$ \\
\hline No. of pathological chest Xrays & $420(76 \%)$ \\
\hline No. of patients with normal baseline chest Xrays which later became abnormal (on day 10) & $36(7.2 \%)$ \\
\hline No. of patients with abnormal baseline chest Xrays which later became normal (on day 10) & $172(34.4 \%)$ \\
\hline Type of involvement at Xray & \\
- consolidation & $261(52.2 \%)$ \\
- bilateral & $164(32.8 \%)$ \\
- unilateral & $97(19.4 \%)$ \\
- patchy GGOs & $301(60.2 \%)$ \\
- pleural effusion. & $180(36 \%)$ \\
\hline $\begin{array}{l}\text { Distribution on baseline Xray } \\
\text { - peripheral predominance }\end{array}$ & $32(6.4 \%)$ \\
- central predominance & \\
- non specific & $196(39.2 \%)$ \\
\hline Distribution on follow up Xrays & $76(15.2 \%)$ \\
- peripheral predominance & $148(29.6 \%)$ \\
\hline - central predominance & \\
- non specific & $130(26 \%)$ \\
\hline No. of pathological Xrays in patients without comorbidities(300) & $48(9.6 \%)$ \\
\hline No. of pathological Xrays in patients with comorbidities(200) & $106(21.2 \%)$ \\
\hline
\end{tabular}


Vaishali Kundu et al; Sch J App Med Sci, Mar, 2021; 9(3): 356-359

\section{DiscuSSION AND CONCLUSION}

The outbreak of COVID-19 pneumonia resulted in a major global health emergency, much larger in comparison to the outbreaks of severe acute respiratory syndrome (SARS) in the year 2003 and Middle East respiratory syndrome (MERS) in the year 2012, both of which were also caused by viruses belonging to the same family coronaviridae. Indeed, COVID-19 pneumonia also shows radiologic similarities to SARS and MERS pneumonia [13].

In our study we included 550 patients who tested positive with real time PCR for COVID-19 during a span of three months. CXRs of 500 patients were properly analysed as CXR of 50 patients were lost due to faulty techniques and errors during data acquisition.

Out of 500 patients, 236(47.2\%) were male and $264(52.8 \%)$ were female. Mean age of patients was 54 years. Co-morbidities were present in 200(40\%) patients including diabetes mellitus (15\%), hypertension $(17 \%)$, asthma $(3.4 \%)$, pulmonary tuberculosis $(2.4 \%)$ and malignancy $(2.2 \%)$ were taken into consideration. These 500 patients had follow up CXRs done at day 10 of testing positive on RTPCR for COVID 19 virus.

$24 \%$ patients had normal chest Xrays while $76 \%$ had pathological chest Xrays. $7.2 \%$ patients had normal baseline chest Xrays which later became abnormal while $34.4 \%$ patients had abnormal baseline chest Xrays which later became normal (on day 10).

$52.2 \%$ patients had consolidation with $32.8 \%$ having bilateral and $19.4 \%$ having unilateral involvement. Patchy ground glass opacities (GGOs), hilar lymphadenopathy and pleural effusion were present in $60.2 \%, 36 \%$ and $6.4 \%$ respectively. $39.2 \%$ patients had peripheral while $15.2 \%$ had central predominance on baseline CXRs. On follow up CXRs $26 \%$ patients had peripheral while $9.6 \%$ had central predominance. $80.6 \%$ of CXRs were pathological in patients without comorbidities and $89 \%$ of CXRs were pathological in patients without comorbidities.

Through this study we have tried to highlight the importance of chest radiography especially portable radiography in inpatient settings in detecting the degree of lung involvement in patients with or without comorbidities

Our study had certain limitations. First, since it was a retrospective study we could not go for follow up for more than 10 days. Second, correlation with CT features could not be done. Third, chest radiographs and RT-PCR testing were dictated by clinical need and time interval between them was not uniform, thus potentially affecting the precision of our analysis.
In comparison to computed tomography portable radiography is inexpensive and much more feasible and thus can be used as the first radiological investigation in COVID-19 patients.

\section{REFERENCES}

1. World Health Organization website. Pneumonia of unknown cause: China. 2020; www.who.int/csr/don/05- january-2020pneumonia-of-unkown-cause-china/ en/. Accessed February 13, 2020

2. World Health Organization website. Coronavirus disease (COVID-19) technical guidance: early investigations. www.who.int/emergencies/diseases/ novel- coronavirus-2019/technicalguidance/earlyinvestigations. Accessed February 15,2020

3. World Health Organization, Novel Coronavirus (2019-nCoV) Situation Report - 11. 2020.https://www.who.int/docs/defaultsource/coronaviruse/situationreports/20200131sitrep-11-ncov.pdf?sfvrsn=de7c0f7_4

4. Zhou S, Wang Y, Zhu T, Xia L. CT Features of Coronavirus Disease 2019 (COVID-19) Pneumonia in 62 Patients in Wuhan, China. Am J Roentgenol. March 2020:1-8. doi:10.2214/AJR.20.22975.

5. Ai T, Yang Z, Hou H. Correlation of Chest CT and RT-PCR Testing in Coronavirus Disease 2019 (COVID-19) in China: A Report of 1014 Cases. Radiology. February 2020:200642. doi:10.1148/radiol.2020200642.

6. Bernheim A, Mei X, Huang M. Chest CT Findings in Coronavirus Disease-19 (COVID-19): Relationship to Duration of Infection. Radiology. February2020:200463. doi:10.1148/radiol.2020200463.

7. Pan F, Ye T, Sun P. Time Course of Lung Changes On Chest CT During Recovery From 2019 Novel Coronavirus (COVID-19) Pneumonia. Radiology. February 2020:200370 doi:10.1148/radiol.2020200370.

8. Ng M-Y, Lee EY, Yang J. Imaging Profile of the COVID-19 Infection: Radiologic Findings and Literature Review. Radiol Cardiothorac Imaging. 2020; 2(1):e200034. doi:10.1148/ryct.2020200034.

9. Chung M, Bernheim A, Mei X. CT Imaging Features of 2019 Novel Coronavirus (2019-nCoV). Radiology. February 2020:200230. doi:10.1148/radiol.2020200230.

10. Chinese Society of Radiology. Chinese Society of Radiology. Radiological diagnosis of new coronavirus infected pneumonitis: Expert recommendation from the Chinese Society of Radiology (First edition). Chin J Radiol. 2020;54(00):E001-E001.

doi:10.3760/cma.j.issn.1005-1201.2020.0001.

11. Zu ZY, Jiang M Di, Xu PP. Coronavirus Disease 2019 (COVID-19): A Perspective from China. Radiology. February 2020:200490. doi:10.1148/radiol.2020200490. 
Vaishali Kundu et al; Sch J App Med Sci, Mar, 2021; 9(3): 356-359

12. ACR Recommendations for the use of Chest Radiography and Computed Tomography (CT) for Suspected COVID-19 Infection|American College of Radiology.https://www.acr.org/Advocacy-andEconomics/ACR-Position-

$\%$ 20Statements/Recommendations-for-Chest-
Radiography-and-CT-for-Suspected-COVID19-

Infection. Accessed March 22, 2020.

13. Ajlan AM, Ahyad RA, Jamjoom LG, Alharthy A, Madani TA. Middle East respiratory syndrome Coronavirus (MERS-CoV) infection: chest CT findings. AJR Am J Roentgenol. 2014;203:782787. 